\title{
MAPA CONCEITUAL COMO FERRAMENTA DE APRENDIZAGEM: REVISÃO INTEGRATIVA DA LITERATURA
}

CONCEPT MAP AS A LEARNING TOOL: AN INTEGRATIVE LITERATURE REVIEW

MAPA CONCEPTUAL COMO HERRAMIENTA DE APRENDIZAJE: UNA REVISIÓN INTEGRADORA DE LA LITERATURA

Jaline Oliveira Medeiros ${ }^{1}$

Rafaella do Carmo Ribeiro ${ }^{2}$

Milena Nunes Alves de Sousa ${ }^{3}$

Palavras-chave:

Metodologia Ativa; Mapa Conceitual;

Aprendizagem.

Keywords:

Active Methodology; Conceptual Map;

Learning.

Palabras clave: Metodología Activa; Mapa Conceptual; Aprendizaje.

Submetido: 02 de Fev. de 2020

Aprovado: 07 de Dez. de 2020

Autor(a) para Correspondência: Milena Nunes Alves de Sousa R. Horácio Nóbrega, $S / N$ Belo Horizonte - Patos, $P B$ CEP:58704-000 E-mail:milenanunes@fiponline.edu.br

\footnotetext{
Certificação de redação científica: E.L.Freire Editora. Edição de texto: Evandro L. Freire. Revisão de provas: Texto definitivo lido e validado pelas autores.
}

\section{RESUMO}

Este estudo teve por objetivou especificar os beneficios do uso do mapa conceitual, para mostrar a relevância dessa estratégia e incentivar seu uso, com base em comprovação científica. Realizou-se uma revisão integrativa da literatura, com levantamento em bases de dados indexadas, como Biblioteca Virtual em Saúde (BVS), Portal de Periódicos da Coordenação de Aperfeiçoamento de Pessoal de Nivel Superior (CAPES) e Scientific Electronic Library Online (SCIELO). O estudo foi realizado em outubro e novembro de 2019, usando os termos de busca "mapa conceitual" e "aprendizagem" para identificar os estudos. Os artigos selecionados indicaram que o uso desses mapas: a) possibilita a organização do conhecimento; b) funciona como recurso de aprendizagem; c) funciona como recurso de hierarquização dos conceitos; e d) promove a autonomia e o pensamento crítico. Desse modo, corrobora-se a importância dessa "nova" estratégia, aplicada por meio de metodologias ativas em instituições de ensino, como instrumento facilitador na aquisição de novos conhecimentos, auxiliando a aprendizagem e a formação profissional dos estudantes.

1. Graduada em Medicina pelo Centro Universitário de Patos (UNIFIP). E-mail: jaline.oliveira@hotmail.com ORCID: $\underline{0000-0002-6524-1361}$

2. Graduada em Medicina pelo UNIFIP. E-mail: rafaellaribeiro_@hotmail.com 0RCID: 0000-0003-1203-6070

3. Pós-Doutora em Promoção de Saúde pela Universidade de Franca (UNIFRAN). Professora no UNIFIP. E-mail: milenanunes@fiponline.edu.br 0RCID: 0000-0001-8327-9147 


\section{ABSTRACT}

This study aimed to specify the benefits of using concept maps to show the relevance of this strategy and to encourage its use, according to scientific evidence. An integrative literature review was carried out, with a survey of indexed databases, such as the Virtual Health Library (VHL), the Journals' Portal of the Brazilian Coordination for the Improvement of Higher Education Personnel (Coordenação de Aperfeiçoamento de Pessoal de Nivel Superior [CAPES]), and the Scientific Electronic Library Online (SCiELO). The study was conducted in October and November 2019, using the search terms 'concept map' and 'learning' to identify the studies. The selected articles showed that the use of these maps: a) enables knowledge organization; b) serves as a learning resource; c) serves as a hierarchy of concepts; and d) promotes autonomy and critical thinking. Thus, the importance of this 'new' strategy is corroborated, it is applied through active methodologies in educational institutions, as a facilitating instrument in the acquisition of new knowledge, aiding students learning and vocational training.

\section{RESUMEN}

Este estudio tuvo como objetivo precisar los beneficios de utilizar mapas conceptuales para mostrar la relevancia de esta estrategia y fomentar su uso, de acuerdo con la evidencia científica. Se realizó una revisión integradora de la literatura, con encuesta en bases de datos indexadas, como la Biblioteca Virtual en Salud (BVS), el Portal de Revistas de la Coordinación Brasileña de Perfeccionamiento del Personal de Educación Superior (Coordenação de Aperfeiçoamento de Pessoal de Nivel Superior [CAPES]) y la Scientific Electronic Library Online (SciELO). El estudio se realizó en octubre y noviembre de 2019, utilizando los términos de búsqueda "mapa conceptual" y "aprendizaje" para identificar los estudios. Los artículos seleccionados mostraron que el uso de estos mapas: a) permite la organización del conocimiento; b) sirve como recurso de aprendizaje; c) sirve como jerarquía de conceptos; y d) promueve la autonomía y el pensamiento crítico. Así, se corrobora la importancia de esta "nueva" estrategia, que se aplica a través de metodologías activas en instituciones educativas, como un instrumento facilitador en la adquisición de nuevos conocimientos, favoreciendo el aprendizaje y la formación profesional de los estudiantes.

\section{INTRODUÇÃ O}

$\mathrm{Na}$ contemporaneidade, busca-se uma transformação paradigmática na metodologia pedagógica, pois os conteúdos ministrados de modo tradicional desencadeiam um comportamento passivo no processo de ensino-aprendizagem por parte dos alunos, que exercem o papel de "depósitos" de informações, sem preocupação com a apropriação de conceitos. Por isso, as mudanças na educação embasadas na Teoria Cognitiva de Aprendizagem têm o intuito de formar indivíduos críticos, reflexivos e criativos para que atuem na formação de seu próprio conhecimento ${ }^{1}$.

Em decorrência disso, algumas estratégias de aprendizagem, dentre elas os mapas conceituais, vêm sendo usadas com o propósito de que os estudantes adquiram novos conceitos de maneira ativa, tornando-se os personagens principais desse evento ${ }^{2}$. Por mapas conceituais se entendem representações esquemáticas do conhecimento, tendo como ponto de partida um conceito-chave e sua correlação com as demais ideias de quem o elabora. Sua estruturação é hierarquizada a partir de palavras principais dentro de pequenas "caixas", seguidas por "linhas" que servem de conectivos para frases explicativas que interligam a ideia ${ }^{3}$.

Propostos pelo pesquisador Joseph Novak, esses diagramas surgiram em meados da década de 1970 como suporte à Teoria da Aprendizagem Significativa de Ausubel e Novak ${ }^{4}$. Ela prevê a aquisição do conhecimento por meio do resgate de conceitos prévios, correlacionando-os com conceitos novos e gerando mudanças na estrutura cognitiva do indivíduo. Com isso, promove-se uma aprendizagem dinâmica e aprende-se um novo conceito ou uma nova proposição capaz de gerar um pensamento crítico sobre determinado tema ${ }^{2}$.

Portanto, reconhecendo que o mapa conceitual funciona como instrumento facilitador na aprendizagem significativa, podendo ser um recurso empregado de várias maneiras no contexto educacional, este estudo teve por objetivo:

- Especificar os benefícios do uso do mapa conceitual como ferramenta de aprendizagem, para mostrar a relevância dessa estratégia e incentivar seu uso, conforme comprovação científica. 


\section{MÉTODO}

Trata-se de uma revisão integrativa da literatura, que se caracteriza pela análise sistemática e ampla de estudos científicos com vistas a promover uma síntese do conhecimento sobre o tema abordado. Além disso, essa metodologia viabiliza a produção de novos estudos com base nas lacunas identificadas durante o processo de pesquisa. Seis fases bem definidas levam à modelagem de cada estudo de acordo com o objetivo almejado e tal método de pesquisa engloba adaptações de suas técnicas, segundo critérios pré-definidos pelo pesquisador ${ }^{5}$.

Inicialmente, esta pesquisa se pautou pela seguinte questão norteadora:

- Quais são os benefícios do uso do mapa conceitual como ferramenta de aprendizagem?

Em seguida, adotaram-se como termos de busca "mapa conceitual" e "aprendizagem" para identificar os estudos, conforme os seguintes critérios de inclusão: a) artigos disponíveis on-line; b) artigos redigidos em português ou inglês; e c) artigos publicados nos últimos dez anos. Já os critérios de exclusão foram: a) publicações repetidas; e b) publicações sem livre acesso on-line; e c) publicações não disponíveis na íntegra on-line.

0 levantamento dos artigos ocorreu em bases de dados indexadas, como Biblioteca Virtual em Saúde (BVS), Portal de Periódicos da Coordenação de Aperfeiçoamento de Pessoal de Nível Superior (CAPES) e Scientific Electronic Library Online (SciELO). Realizado em outubro e novembro de 2019, o estudo identificou 122 artigos, sendo considerados elegíveis 27. Ao aplicar os critérios de inclusão e exclusão, 10 artigos foram selecionados e submetidos à leitura na íntegra.

0 processo de seleção dos artigos é detalhado na Figura 1.

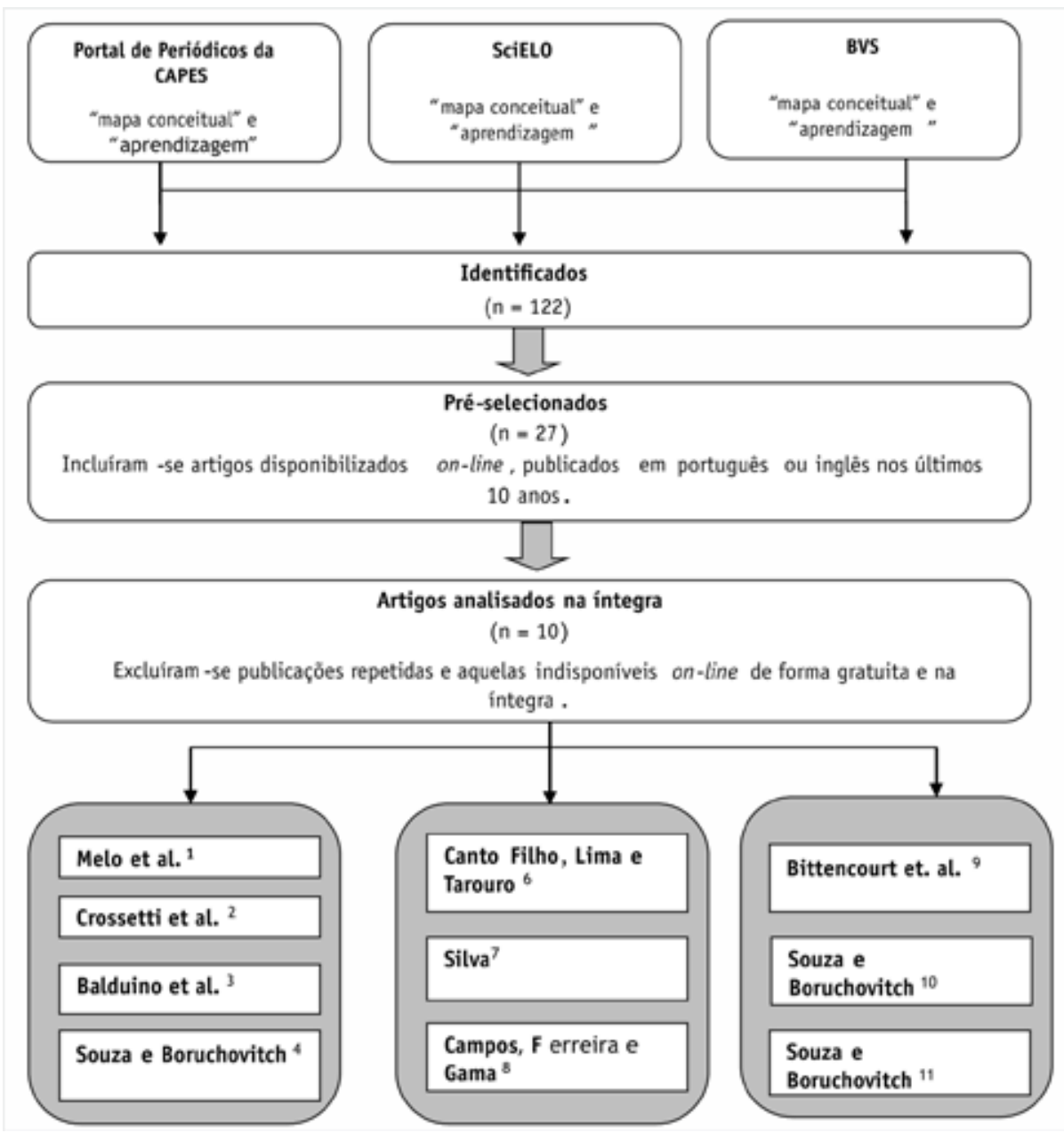

Figura 1. Fluxograma do processo de seleção dos artigos incluídos na revisão integrativa da literatura. Fonte: Elaborado pelas autoras. 
0 passo seguinte foi a análise dos artigos selecionados por meio de uma planilha do Microsoft Word, organizada por nome do(s) autor(es), ano, periódico, portal/base de dados e idioma. Essa planilha serviu como guia para a categorização dos estudos em 5 temas principais no estudo com mapas conceituais: a) Recurso de aprendizagem; b) Organização do conhecimento; c) Hierarquização dos conceitos; d) Autonomia; e e) Desenvolvimento do pensamento crítico.

A conclusão de todas as fases do método adotado viabilizou a revisão e discussão dos resultados para, enfim, apresentar uma sintese do conhecimento sobre o tema abordado.

\section{RESULTADOS}

Quanto ao ano de publicação dos estudos selecionados, a amostra final de 10 artigos variou de 2009 a 2017: a) 3 deles vieram a lume em 2010; b) 2 foram publicados em 2013 e outros 2 em 2015; e c) 1 artigo foi publicado nos anos de 2009, 2014 e 2017.

No que diz respeito às bases de dados: a) 5 estudos foram selecionados na BVS; b) 4 vieram do Portal de Periódicos da CAPES; e c) 1 estudo foi disponibilizado pela SciELO.

Em relação ao idioma: a) 8 artigos selecionados foram redigidos em língua portuguesa; e b) 2 foram redigidos em língua inglesa.

Por fim, os periódicos que publicaram os artigos são diversificados.

0 Quadro 1 ilustra a análise dos artigos selecionados na planilha do Microsoft Word.

Quadro 1 - Distribuição dos artigos selecionados para a revisão integrativa da literatura

\begin{tabular}{|c|c|c|c|}
\hline Título & Periódico & Base & Idioma \\
\hline $\begin{array}{l}\text { Diálogo sobre a construção de um mapa conceitual } \\
\text { como recurso para aprendizagem: relato de } \\
\text { experiencia }{ }^{1}\end{array}$ & $\begin{array}{c}\text { Revista Iberoamericana } \\
\text { de Educación e } \\
\text { Investigación en } \\
\text { Enfermería }\end{array}$ & BVS & Português \\
\hline $\begin{array}{l}\text { Estratégias de ensino das habilidades do } \\
\text { pensamento crítico na enfermagem }{ }^{2}\end{array}$ & $\begin{array}{l}\text { Revista Gaúcha de } \\
\text { Enfermagem }\end{array}$ & BVS & Português \\
\hline $\begin{array}{l}\text { Utilization of a concept maps in order to study a } \\
\text { methodological foundation: experience account }{ }^{3}\end{array}$ & Ciência, Cuidado e Saúde & BVS & Inglês \\
\hline $\begin{array}{l}\text { Mapa conceitual: seu potencial como instrumento } \\
\text { avaliativo }{ }^{4}\end{array}$ & Pro-Posições & CAPES & Português \\
\hline $\begin{array}{l}\text { Mapas conceituais de projeto: uma ferramenta } \\
\text { para projetar objetos de aprendizagem } \\
\text { significativa }{ }^{6}\end{array}$ & $\begin{array}{l}\text { Ciência \& Educação } \\
\text { (Bauru) }\end{array}$ & SciELO & Português \\
\hline $\begin{array}{l}\text { Mapas conceituais: propostas de aprendizagem e } \\
\text { avaliação } 0^{7}\end{array}$ & $\begin{array}{c}\text { Administração Ensino e } \\
\text { Pesquisa }\end{array}$ & CAPES & Português \\
\hline $\begin{array}{l}\text { Mapa conceitual: ferramenta didática no curso de } \\
\text { Fonoaudiologia }{ }^{8}\end{array}$ & $\begin{array}{l}\text { Distúrbios da } \\
\text { Comunicação }\end{array}$ & BVS & Português \\
\hline $\begin{array}{l}\text { Concept maps of the graduate programme in } \\
\text { nursing: experience report }{ }^{9}\end{array}$ & $\begin{array}{l}\text { Revista Gaúcha de } \\
\text { Enfermagem }\end{array}$ & BVS & Inglês \\
\hline $\begin{array}{l}\text { Mapas conceituais e avaliação formativa: tecendo } \\
\text { aproximações }{ }^{10}\end{array}$ & Educação e Pesquisa & CAPES & Português \\
\hline $\begin{array}{l}\text { Mapas conceituais: estratégia de ensino/ } \\
\text { aprendizagem e ferramenta avaliativa }{ }^{11}\end{array}$ & Educação em Revista & CAPES & Português \\
\hline
\end{tabular}

Fonte: Elaborado pelas autoras.

Após classificar os artigos em 5 categorias, identificou-se que: a) 8 estudos corresponderam à categoria 2 (Organização do conhecimento); b) 7 abordaram igualmente as categorias 1 e 3 (Recurso de aprendizagem 
e Hierarquização dos conceitos, respectivamente); e c) 6 estudos integraram as categorias 4 (Autonomia) e 5

(Desenvolvimento do pensamento crítico).

Esses achados de pesquisa são descritos no Quadro 2.

Quadro 2 - Categorização dos artigos selecionados para a revisão integrativa da literatura

Categoria 1 - Recurso de aprendizagem

Diálogo sobre a construção de um mapa conceitual como recurso para aprendizagem: relato de experiência ${ }^{1}$

Mapa conceitual: seu potencial como instrumento avaliativo ${ }^{4}$

Mapas conceituais de projeto: uma ferramenta para projetar objetos de aprendizagem significativa ${ }^{6}$

Mapas conceituais: propostas de aprendizagem e avaliação ${ }^{7}$

Mapa conceitual: ferramenta didática no curso de Fonoaudiologia ${ }^{8}$

Mapas conceituais e avaliação formativa: tecendo aproximações ${ }^{10}$

Mapas conceituais: estratégia de ensino/aprendizagem e ferramenta avaliativa ${ }^{11}$

\section{Categoria 2 - Organização do conhecimento}

Diálogo sobre a construção de um mapa conceitual como recurso para aprendizagem: relato de experiência ${ }^{1}$ Utilization of a concept maps in order to study a methodological foundation: experience account ${ }^{3}$

Mapa conceitual: seu potencial como instrumento avaliativo ${ }^{4}$

Mapas conceituais: propostas de aprendizagem e avaliação ${ }^{7}$

Mapa conceitual: ferramenta didática no curso de Fonoaudiologia ${ }^{8}$

Concept maps of the graduate programme in nursing: experience report ${ }^{9}$

Mapas conceituais e avaliação formativa: tecendo aproximações ${ }^{10}$

Mapas conceituais: estratégia de ensino/aprendizagem e ferramenta avaliativa ${ }^{11}$

\section{Categoria 3 - Hierarquização dos conceitos}

Diálogo sobre a construção de um mapa conceitual como recurso para aprendizagem: relato de experiência ${ }^{1}$

Estratégias de ensino das habilidades do pensamento crítico na enfermagem ${ }^{2}$

Utilization of a concept maps in order to study a methodological foundation: experience account ${ }^{3}$

Mapa conceitual: seu potencial como instrumento avaliativo ${ }^{4}$

Mapas conceituais: propostas de aprendizagem e avaliaçã ${ }^{7}$

Mapas conceituais e avaliação formativa: tecendo aproximações ${ }^{10}$

Mapas conceituais: estratégia de ensino/aprendizagem e ferramenta avaliativa ${ }^{11}$

\section{Categoria 4 - Autonomia}

Diálogo sobre a construção de um mapa conceitual como recurso para aprendizagem: relato de experiência ${ }^{1}$

Mapa conceitual: seu potencial como instrumento avaliativo ${ }^{4}$

Mapas conceituais: propostas de aprendizagem e avaliaçã ${ }^{7}$

Mapa conceitual: ferramenta didática no curso de Fonoaudiologia ${ }^{8}$

Mapas conceituais e avaliação formativa: tecendo aproximações ${ }^{10}$

Mapas conceituais: estratégia de ensino/aprendizagem e ferramenta avaliativa ${ }^{11}$

\section{Categoria 5 - Desenvolvimento do pensamento crítico}

Diálogo sobre a construção de um mapa conceitual como recurso para aprendizagem: relato de experiencia ${ }^{1}$

Estratégias de ensino das habilidades do pensamento crítico na enfermagem ${ }^{2}$

Mapa conceitual: seu potencial como instrumento avaliativo ${ }^{4}$

Mapas conceituais de projeto: uma ferramenta para projetar objetos de aprendizagem significativa ${ }^{6}$

Mapa conceitual: ferramenta didática no curso de Fonoaudiologia ${ }^{8}$

Concept maps of the graduate programme in nursing: experience report ${ }^{9}$

Fonte: Elaborado pelas autoras. 


\section{DISCUSSÃO}

Diante da aplicação de métodos inovadores em instituições de ensino que usam metodologias ativas, observou-se que a prática pedagógica vai muito além do limite técnico, podendo alcançar a ação-reflexãoação. Nesse caso, os mapas conceituais se mostraram uma boa ferramenta de aprendizagem ${ }^{12}$.

Observou-se que 0 uso dessa estratégia no processo de aprendizagem apresenta diversas benesses que podem contribuir significativamente na formação de profissionais crítico-criativos, capacitados para solucionar problemas e demandas, superando o modelo tradicional de memorização e verificação de conteúdo.

Desse modo, as principais vantagens foram classificadas em 5 categorias: a) Recurso de aprendizagem ${ }^{1,4,6-8,10,11}$; b) Organização do conhecimento $0^{1,4,7-11}$; c) Hierarquização dos conceitos $^{1-3,4,7,10,11}$; d) Autonomia' $1,4,7,8,10,11$; e e) Desenvolvimento do pensamento crítico ${ }^{1,3,4,6,8,9}$.

Estudos ${ }^{8,13}$ defendem o uso do mapa conceitual como recurso de aprendizagem por agregarem informações, selecionando e simplificando os elementos mais importantes do assunto abordado, tornando a memorização mais efetiva, quando comparada com listas lineares de informações. Também se observou a ampliação do pensamento durante a elaboração do mapa, pois ele estimula os estudantes a identificarem relações entre os conceitos.

Em um estudo comparativo entre alunos que usaram os mapas e alunos que tiveram aula segundo os métodos tradicionais, o primeiro grupo atingiu níveis mais profundos de discussão, mostrando que a aprendizagem foi mais significativa ${ }^{13}$.

Complementando a ideia, uma pesquisa explica que os mapas conceituais se mostraram uma boa ferramenta de aprendizagem por conterem palavraschave que indicam a ideia principal e sua relação com as demais proposições, criando uma conexão sequencial acerca do tema abordado ${ }^{1}$. Além de funcionar como um recurso de aprendizagem, também é possível proporcionar a organização do conhecimento a partir da construção de uma rede semântica, capaz de gerar compreensão e solidificação do estudo ${ }^{10}$.

Em contrapartida, observou-se que o método tradicional de ensino-aprendizagem prioriza a memorização e a fixação de conteúdos com sua exposição, algo diferente dos mapas conceituais, que proporcionam a internalização e a aplicação dos

\section{...ele estimula \\ os estudantes \\ a identificarem \\ relações entre os conceitos}

conceitos, desde que sejam estruturados e aplicados com algum objetivo, como: a) resolver problemas; b) correlacionar ideias; c) sedimentar conteúdos; e d) integrar conteúdos ${ }^{7}$.

Um ponto fundamental dessa estratégia é a possibilidade do aprendiz representar o conteúdo incorporado em sua estrutura cognitiva sem importar se está errado ou correto, mas com o intuito principal de revelar se há evidências de que a aprendizagem ocorreu de modo significativo, isto é, se os conceitos prévios sofreram alterações diante dos novos ${ }^{11}$. Portanto, os mapas conceituais demonstram uma "nova" maneira de organizar, estruturar e hierarquizar os assuntos, por meio da organização cognitiva daqueles que os desenvolvem?

Nesse caso, outra característica importante sobre esses diagramas foi sua capacidade de hierarquizar conceitos, ou seja, iniciam-se com aspectos mais gerais e essenciais e culminam em pontos mais específicos e secundários. Essa configuração é respaldada porduas hipóteses: a) a primeira indica que a melhor maneira de absorver conteúdo é tendo como ponto de partida informações mais amplas e gerais, seguindo para ramificações menos abrangentes; e b) a segunda indica que a aprendizagem ocorre pela ordem sequencial das proposições com base na estrutura cognitiva do estudante ${ }^{3,11}$.

Entretanto, colocar em prática a composição vertical e descendente do mapa nem sempre será uma tarefa fácil para os estudantes. Como visto em outra investigação ${ }^{4}$, os alunos apresentaram dificuldade para criar hierarquias, pois era necessário identificar o principal conceito e saber qual conceito era mais geral e inclusivo. Além disso, os problemas continuaram, pois todos os demais pareciam igualmente importantes.

Em outra perspectiva, no momento em que o ensino-aprendizagem passa a ser centrado na pessoa, isso proporciona autonomia ao aluno, pois a incorporação do conhecimento decorre da participação ativa do indivíduo em sua formação ${ }^{12}$. 
Desse modo, o estudante desenvolve a competência de meta-aprendizagem, ou seja, "aprender a aprender", durante esse processo participativo ${ }^{14}$.

Ainda acerca desse assunto, durante o processo de construção dos mapas, os questionamentos e desafios propostos pelo facilitador contribuíram para que os alunos percebessem que o saber depende diretamente do autoestudo, desenvolvido por meio da aprendizagem ativa e da iniciativa de buscar respostas, tornando o conhecimento mais duradouro e sólido ${ }^{1}$. Um estudo aponta que: “o processo de ensino estabelece uma relação diferenciada com o educando, onde se observa uma trajetória de construção do saber e promoção da aprendizagem"15:146.

Apesar da apresentação de um novo recurso de ensino parecer confuso ou difícil para os estudantes, inicialmente, durante a discussão com o facilitador, essa ideia vai sendo diluída ${ }^{1}$. A partir disso, os alunos começam a ver a possibilidade de aprender a aprender, compreendendo que a forma como vinham estudando não estimulava em nada o pensamento crítico e reflexivo. Esse método ativo tem como princípio teórico a autonomia do aluno, que Paulo Freire destaca ${ }^{16}$.

As representações esquemáticas proporcionam melhor visualização do tema, deixando o indivíduo ciente da ideia principal, constituindo uma ferramenta capaz de desenvolver o pensamento crítico pelo poder de síntese, discussão, reflexão, criação e compreensão que essa estratégia proporciona ${ }^{4}$.

Outro estudo ${ }^{2}$ aborda o pensamento crítico em profissionais da saúde como um importante auxílio em suas decisões diante dos pacientes. Para esses profissionais, o pensamento crítico se define como algo cuidadoso e focalizado em resultados que requer um pensar motivado pelas necessidades do paciente, da família e da comunidade. Portanto, relacioná-lo à base de conhecimentos que orienta as ações desses profissionais poderia melhorar significativamente os cuidados prestados.

0 desenrolar do pensamento crítico também advém da observação dos erros, pois os mapas envolvem a possibilidade de fazer comparações com os elaborados por outros colegas, algo diferente de uma descrição linear. Desse modo, os estudantes aprendem com o reconhecimento do erro, gerando maior esforço para a correção e a aprendizagem ${ }^{4}$.

Vale ressaltar, ainda, que as habilidades de pensamento crítico e raciocínio lógico são passíveis de aprendizado e aprimoramento. Para tanto, a aprendizagem precisa fazer parte de um processo

\section{...Constituindo uma ferramenta capaz de desenvolver o pensamento crítico...}

contínuo e dinâmico para proporcionar a aquisição de novos conhecimentos ${ }^{6}$.

Finalmente, identificou-se como limitação desta revisão integrativa da literatura o uso de artigos publicados há mais de cinco anos, devido à escassez de estudos mais atualizados que respondessem à questão norteadora e que se adequassem aos critérios de inclusão. De todo modo, acredita-se que as informações deste estudo se mostram pertinentes diante de seu objetivo, corroborando a relevância do tema para a população e as instituições de ensino, a fim de estimular o uso dessa nova ferramenta no processo de aprendizagem.

\section{CONCLUSÃO}

Este estudo identificou 5 categorias para descrever os benefícios do uso do mapa conceitual como ferramenta no processo de aprendizagem a) Recurso de aprendizagem; b) Organização do conhecimento; c) Hierarquização dos conceitos; d) Autonomia; e e) Desenvolvimento do pensamento crítico.

Observou-se que tais categorias se encontram correlacionadas, pois a existência de uma proporciona a ocorrência das outras. Desse modo, corrobora-se a importância da aplicabilidade desta "nova" estratégia, que vem sendo aplicada mediante metodologias ativas em instituições de ensino, como instrumento facilitador na aquisição de novos conhecimentos, auxiliando a aprendizagem e a formação profissional dos estudantes.

\section{CONTRIBUIÇÃO DAS AUTORAS}

Jaline Oliveira Medeiros e Rafaella do Carmo Ribeiro colaboraram com a realização da pesquisa, o delineamento do estudo e a redação do manuscrito. Milena Nunes Alves de Sousa colaborou com o delineamento do estudo e a redação e revisão crítica do manuscrito. 


\section{REFERÊNCIAS}

1. Melo LAA, Bezerra MVM, Melo LA, Martins CMA, Correia MS, Albuquerque RS. Diálogo sobre a construção de um mapa conceitual como recurso para aprendizagem: relato de experiencia. Rev Iberoam Educ Invest Enferm [serial on the internet]. 2015 [cited 2020 Dec 14];5(4):50-8. Available from: https://www.enfermeria21.com/revistas/aladefe/ articulo/184/dialogo-sobre-a-construcao-de-ummapa-conceitual-como-recurso-para-aprendizagemrelato-de-experiencia/

2. Crossetti MG0, Bittencourt GKGD, Schaurich D, Tanccini T, Antunes M. Estratégias de ensino das habilidades do pensamento crítico na enfermagem. Rev Gaúch Enferm [serial on the internet]. 2009 [cited 2020 Dec 14];30(4):732-41. Available from: https://www.scielo.br/pdf/rgenf/v30n4/a21v30n4. pdf

3. Balduino AFA, Gomes IM, Lacerda MR, Mantovani MF. Utilization of a concept maps in order to study a methodological foundation: experience account. Ciênc Cuid Saúde [serial on the internet]. 2013 [cited 2020 Dec 14];12(1):177-83. Available from: http://www.revenf.bvs.br/pdf/ccs/v12n1/23.pdf

4. Souza NA, Boruchovitch E. Mapa conceitual: seu potencial como instrumento avaliativo. ProPosições [serial on the internet]. 2010 [cited 2020 Dec 14];21(3):173-92. Available from: https://www. scielo.br/pdf/pp/v21n3/v21n3a11.pdf

5. Moreira MADMD, Lustusa AM, Dutra F, Barros E0, Batista JBV, Duarte MCS. Políticas públicas de humanização: revisão integrativa da literatura. Ciênc Saúde Colet [serial on the internet]. 2015 [cited 2020 Dec 14];20(10):3231-42. Available from: https://www.scielo.br/pdf/csc/v20n10/1413-8123csc-20-10-3231.pdf

6. Canto Filho AB, Lima JV, Tarouco LMR. Mapas conceituais de projeto: uma ferramenta para projetar objetos de aprendizagem significativa. Ciênc Educ (Bauru) [serial on the internet]. 2017 [cited 2020 Dec 14];23(3):723-40. Available from: https://www.scielo.br/pdf/ciedu/v23n3/1516-7313ciedu-23-03-0723.pdf

7. Silva EC. Mapas conceituais: propostas de aprendizagem e avaliação. Administração Ensino e Pesquisa [serial on the internet]. 2015 [cited 2020 Dec 14];16(4):785-815. Available from: https:// raep.emnuvens.com.br/raep/article/view/385/196

8. Campos NF, Ferreira JM, Gama ACC. Mapa conceitual: ferramenta didática no curso de Fonoaudiologia. Distúrbios da Comunicação [serial on the internet]. 2014 [cited 2020 Dec 14];26(1):1107. Available from: https://revistas.pucsp.br/index. $\mathrm{php/dic/article/view/13937/14179}$
9. Bittencourt GKGD, Nóbrega MMI, Medeiros ACT, Furtado LG. Concept maps of the graduate programme in nursing: experience report. Rev Gaúch Enferm [serial on the internet]. 2013 [cited 2020 Dec 14];34(2):172-6. Available from: https://www. scielo.br/pdf/rgenf/v34n2/v34n2a22.pdf

10. Souza NA, Boruchovitch E. Mapas conceituais e avaliação formativa: tecendo aproximações. Educação e Pesquisa [serial on the internet]. 2010 [cited 2020 Dec 14];36(3):795-810. Available from: https://www.scielo.br/pdf/ep/v36n3/v36n3a10.pdf

11. Souza NA, Boruchovitch E. Mapas conceituais: estratégia de ensino/aprendizagem e ferramenta avaliativa. Educ Rev [serial on the internet]. 2010 [cited 2020 Dec 14];26(3):195-218. Available from: https://www.scielo.br/pdf/edur/v26n3/v26n3a10. pdf

12. Macedo KDS, Acosta BS, Silva EB, Souza NS, Beck CLC, Silva KKD. Metodologias ativas no ensino em saúde. Esc Anna Nery Rev Enferm [serial on the internet]. 2018 [cited 2020 Dec 14];22(3):19. Available from: https://www.scielo.br/pdf/ean/ v22n3/pt 1414-8145-ean-22-03-e20170435.pdf

13. Carvalho DPSRP, Vitor AF, Barichello E, Villar RLA, Santos VEP, Ferreira Jr MA. Aplicação do mapa conceitual: resultados com diferentes métodos de ensino-aprendizagem. Aquichan [serial on the internet]. 2016 [cited 2020 Dec 14];16(3):382-91. Available from: http://www.scielo.org.co/pdf/aqui/ v16n3/v16n3a09.pdf

14. Barreto IDP, Gomes PA, Furlaneto IP, Barreto B. Avaliação das estratégias de autoaprendizagem em alunos de um curso de medicina em Belém - Pará. Rev Bras Educ Méd [serial on the internet]. 2019 [cited 2020 Dec 14];43(4):36-36. Available from: https://www.scielo.br/pdf/rbem/v43n4/1981-5271rbem-43-4-0036.pdf

15. Paiva MRF, Parente JRF, Brandão IR, Queiroz AHB. Metodologias ativas de ensino-aprendizagem: revisão integrativa. Sanare (Sobral, Online) [serial on the internet]. 2016 [cited 2020 Dec 14];15(2):145-53. Available from: https://sanare.emnuvens.com.br/ sanare/article/view/1049/595

16. Xavier LN, Oliveira GL, Gomes AA, Machado MFAS, Eloia SMC. Analisando as metodologias ativas na formação dos profissionais de saúde: uma revisão integrativa. Sanare (Sobral, Online) [serial on the internet]. 2014 [cited 2020 Dec 14];13(1):76-88. Available from: https://sanare.emnuvens.com.br/ sanare/article/view/436/291

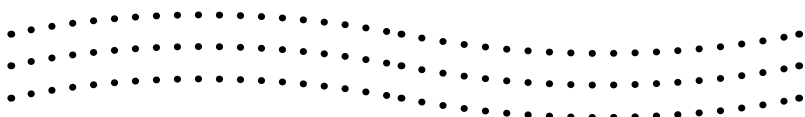

